\title{
Trends in Food Insecurity in the USA for Individuals with Prediabetes, Undiagnosed Diabetes, and Diagnosed Diabetes
}

\author{
Rebekah J. Walker, $P h D^{1,2}$, Jessica Grusnick, PA-C ${ }^{1,2}$, Emma Garacci, $\mathrm{MS}^{1,2}$, Carlos Mendez, MD ${ }^{1,3}$, \\ and Leonard E. Egede, MD, MS ${ }^{1,2}$
}

'Division of General Internal Medicine, Department of Medicine, Froedtert \& The Medical College of Wisconsin, Milwaukee, WI, USA; ${ }^{2}$ Center for Advancing Population Science, Medical College of Wisconsin, Milwaukee, WI, USA; ${ }^{3}$ Division of Diabetes and Endocrinology, Clement J. Zablocki VA Medical Center, Milwaukee, WI, USA. KEY WORDS: food insecurity; diabetes; undiagnosed diabetes;
prediabetes; trends.

$\mathrm{J}$ Gen Intern Med 34(1):33-5

DOI: $10.1007 / \mathrm{s} 11606-018-4651-\mathrm{z}$

๑) Society of General Internal Medicine 2018

\section{INTRODUCTION}

Approximately $12 \%$ of the adult population have diabetes, with nearly 1 in 4 being undiagnosed [1]. Additionally, 84.1 million US adults have prediabetes [1]. A healthy diet is important for disease management, both to promote weight loss and to prevent progression or complications [1]. However, social factors influencing diet, such as food security, can impact an individual's ability to manage or delay disease [2]. Food insecurity indicates either a lack of availability or a lack of ability to acquire healthy food [3].

While food insecurity in the overall population has decreased since 2011, in those diagnosed with cardiometabolic diseases, it has continued to climb [2]. In addition, little research on food insecurity has been conducted in populations with undiagnosed diabetes or prediabetes. Therefore, the aim of this paper was to investigate trends in food insecurity for those with diagnosed diabetes, undiagnosed diabetes, and prediabetes using nationally representative data from 2005 to 2014.

\section{METHODS}

National Health and Nutrition Examination Survey (NHANES) survey data from 2005 to 2014 was analyzed: 27,218 adults aged 20 and over (representing a US population of 217,916,246) were included [4].

Food insecurity during the prior year was based on response to the Food Security Survey Module [3]. Questions ask whether individuals had enough money to purchase food, skipped meals due to cost, or ate less because of cost [3]. Full and marginal food security were considered food secure, while low and very low food security were considered food insecure.

Diabetes status was based on response to diabetes-related questions and HbAlc. Diagnosed diabetes included those

Published online September 13, 2018 answering "yes" to having been told by a health professional they had diabetes, taking insulin, or taking diabetic pills to lower blood sugar. Undiagnosed diabetes included those who answered "no" to all three questions but had an HbAlc $\geq$ $6.5 \%$. Prediabetes included those who answered "yes" to having been told they had prediabetes or had $5.7 \% \leq \mathrm{HbA} 1 \mathrm{c}$ $<6.5 \%$.

Analyses accounted for complex survey design including oversampling, nonresponse, and post-stratification using SAS v9.4. Weighted percent reporting food insecurity by diabetes status over survey wave was compared using a CochranArmitage trend test. Logistic regression models for food insecurity by diabetes status were adjusted by survey wave and demographic variables.

\section{RESULTS}

$18.2 \%$ of participants reported food insecurity between 2005 and 2014. Food insecurity increased from $9.7 \%$ in 2005-2006 to $15.2 \%$ in 2013-2014 ( $p<0.001)$ (see panel 1 of Fig. 1). The average annual rate change was $1.6 \%$ (95\% CI $0.5-2.6 \%$ ), representing significant year-over-year increase. Panel 2 of Figure 1 shows trends by diabetes status, with significant differences in weighted prevalence $(p<0.001)$. Prevalence of food insecurity increased more dramatically for those with undiagnosed diabetes than other groups.

Table 1 shows that after adjusting for demographics, compared to individuals with no diabetes, the odds ratio for food insecurity for prediabetes is 1.39 (95\% CI $1.21-1.59)$, for undiagnosed diabetes is 1.81 (95\% CI 1.37-2.38), and for diagnosed diabetes is 1.58 (95\% CI 1.29-1.93).

\section{DISCUSSION}

Between 2005 and 2014, compared to individuals without diabetes, those with prediabetes were $39 \%$ more likely to be food insecure, those with diagnosed diabetes were $58 \%$ more likely to be food insecure, and those with undiagnosed diabetes were $81 \%$ more likely to be food insecure.

Given the confluence of factors that impact low-income individuals [5], the increase in overall prevalence by diabetes 

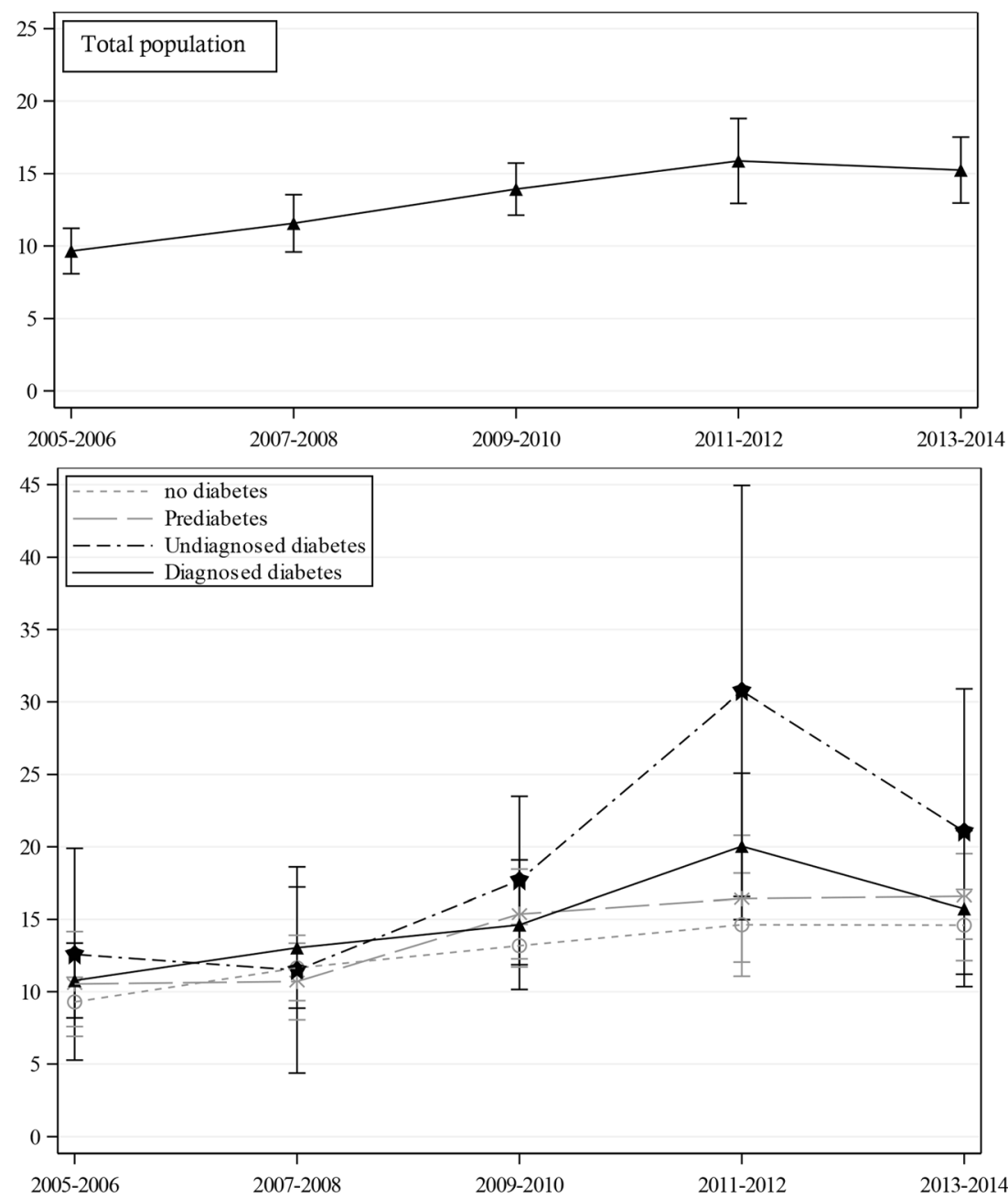

Figure 1 Trends in food insecurity 2005-2014, \% reporting food insecurity overall and by diabetes status.

status and increased odds for prediabetes or undiagnosed diabetes has important implications for primary care. As individuals with lower socioeconomic status have limited access to the healthcare system [5], increasing diabetes screening in food insecure populations is needed. Offering community screening events or developing methods for engaging food insecure populations in primary care may help improve diagnosis and overall health. In addition, because adherence to a healthy diet can help prevent transition from prediabetes to diabetes, the increased existence of food insecurity in those with prediabetes is worth noting. Screening individuals with prediabetes for food insecurity may help inform medical recommendations to help reverse or slow progression to diabetes. Barriers to a healthy diet noted by low-income individuals with diabetes include the need focus on understanding of concepts and definitions, such as nutritional terms or nutrition labels [6]. These factors should be taken into account in the clinical encounter to better align recommendations with socioeconomic realities.

Table 1 Multivariable Logistic Regression Estimates for Food Insecurity by Diabetes Status

\begin{tabular}{|c|c|c|c|c|}
\hline & Crude odds ratio OR $(95 \% \mathrm{CI})$ & $p$ value & Adjusted odds ratio* OR (95\% CI) & $p$ value \\
\hline Diabetes status & & 0.0003 & & $<0.0001$ \\
\hline No diabetes & Reference & & Reference & \\
\hline Prediabetes & $1.15(1.01-1.31)$ & & $1.39(1.21-1.59)$ & \\
\hline Undiagnosed diabetes & $1.61(1.26-2.07)$ & & $1.81(1.37-2.38)$ & \\
\hline Diagnosed diabetes & $1.23(1.04-1.46)$ & & $1.58(1.29-1.93)$ & \\
\hline Survey year & & $<0.0001$ & & 0.0006 \\
\hline $2005-2006$ & Reference & & Reference & \\
\hline $2007-2008$ & $1.22(0.96-1.57)$ & & $1.09(0.87-1.38)$ & \\
\hline $2009-2010$ & $1.52(1.22-1.89)$ & & $1.38(1.12-1.70)$ & \\
\hline 2011-2012 & $1.77(1.35-2.31)$ & & $1.51(1.20-1.90)$ & \\
\hline 2013-2014 & $1.68(1.33-2.13)$ & & $1.55(1.23-1.96)$ & \\
\hline
\end{tabular}

*Adjusted models include gender, age, race, education level, marital status, ratio of family income to poverty level, and survey year 
Corresponding Author: Leonard E. Egede, MD, MS; Division of General Internal Medicine, Department of Medicine Froedtert \& The Medical College of Wisconsin, 9200 West Wisconsin Avenue, Milwaukee, WI 53226-3596, USA (e-mail: legede@mcw.edu).

Authors' Contributions LEE obtained funding for the study. LEE, $R J W$, and EG designed the study. EG acquired and analyzed the data. RJW, JG, CM, and LEE interpreted the data. RJW, JG, EG, CM, and LEE drafted the manuscript, critically revised the manuscript for intellectual content, and approved the final manuscript.

Funding This study was supported by the National Institute of Diabetes and Digestive and Kidney Diseases (grant K24DK093699, Principal Investigator: Leonard Egede, MD).

\section{Compliance with Ethical Standards:}

Conflict of Interest: The authors declare that they do not have a conflict of interest.

\section{REFERENCES}

1. Centers for Disease Control and Prevention (CDC). National Diabetes Statistics Report, 2017. Atlanta, GA: U.S. Department of Health and Human Services; 2017.

2. Berkowitz, S.A., et al., Trends in food insecurity for adults with cardiometabolic disease in the United States: 2005-2012. PLoS ONE, 2017. 12(6): p. e0179172.

3. Coleman-Jensen A, R.M., Gregory C, Singh A, Household food security in the United States in 2016. Economic Research Report No. (ERR-237) 44 pp, 2017.

4. Centers for Disease Control and Prevention (CDC). National Center for Health Statistics (NCHS). National Health and Nutrition Examination Survey Data. Hyattsville, MD: U.S. Department of Health and Human Services, Centers for Disease Control and Prevention, 2017 https://www. cdc.gov/nchs/nhanes/index.htm. Accessed July 24, 2018.

5. Shi L and Stevens GD. Vulnerability and unmet health care needs: the influence of multiple risk factors. JGIM 2005; 20: 148-154.

6. Gazmararian JA, Ziemer DC, Barnes C. Perception of barriers to self-care management among diabetic patients. Diabetes Educ. 2009;35(5):778888 . 